\title{
Single and combined effect of high- frequency loading and bisphosphonate treatment on the bone micro-architecture of ovariectomized rats
}

\author{
K. Hatori ${ }^{1,2}$, G. V. Camargos ${ }^{1,3}$, M. Chatterjee ${ }^{1}$, F. Faot ${ }^{1,4}$, K. Sasaki $^{2}$, J. Duyck ${ }^{1}$ and K. Vandamme ${ }^{1}$
}

(1)

Department of Oral Health Sciences, BIOMAT Research Group, KU Leuven \& University Hospitals Leuven, Leuven, Belgium

(2)

Division of Advanced Prosthetic Dentistry, Tohoku University Graduate School of Dentistry, Sendai, Japan

(3)

Piracicaba Dental School, Department of Prosthodontics and Periodontology, State University of Campinas, Piracicaba, São Paulo, Brazil

(4)

Department of Restorative Dentistry, Prosthodontics Division, School of Dentistry, Federal University of Pelotas, Pelotas, Rio Grande do Sul, Brazil

\section{K. Vandamme}

Email: Katleen.Vandamme@med.kuleuven.be

Received: 20 January 2014Accepted: 13 August 2014Published online: 19 September 2014

Abstract

Summary

Mechanical loading at high frequency affects bone. Whether this also applies to osteoporotic bone, combined or not with bisphosphonate therapy, was investigated in this animal study through imaging. An anabolic effect of high-frequency loading on osteoporotic bone, however non-synergistic with bisphosphonates, was found, thereby revealing its potential for treatment of osteoporosis.

Introduction

In an effort to elucidate the effect of high-frequency (HF) loading on bone and to optimize its potential for treatment osteoporosis, this study aimed to investigate the effect of HF loading via whole body vibration (WBV), alone or in association with bisphosphonate treatment (alendronate-ALN), on the micro-architecture of ovariectomy (OVX)-induced compromised bone.

Methods

Eighty-four female Wistar rats were ovariectomized (OVX) or sham-operated (shOVX). OVX animals were treated either with ALN (3 days/week at a dose of $2 \mathrm{mg} / \mathrm{kg}$ ) or with saline solution. Each group (shOVX, OVX, ALN) was further divided into subgroups relative to the loading status (sham-WBV versus WBV) and the duration of experimental period (4 days versus 14 days). (Sham)WBV loading was applied for $10 \mathrm{~min} /$ day using 10 consecutive steps of HF loading (130, 135, 140, 145, 150, 130, 135, 140, 145, $150 \mathrm{~Hz})$. Tibial bone structural 
responses to WBV and/or ALN treatment were analyzed using ex vivo micro-computed tomography.

Results

The animal's hormonal status displayed a major impact on the trabecular and cortical bone structural parameters. Furthermore, mechanical treatment with HF WBV increased the cortical thickness and reduced the medullar area in OVX rats. However, OVX trabecular bone was not affected by HF stimuli. Finally, ALN prevented OVX-associated bone loss, but the association of ALN with WBV did not lead to a synergistic bone response in OVX bone. Conclusions

HF WBV mechanical stimulation displayed an anabolic effect on osteoporotic cortical bone, confirming its therapeutic properties for enhancing compromised bone. Additionally, its association with bisphosphonates' administration did not produce any additive effect on the bone micro-architecture in the present study.

Electronic supplementary material

The online version of this article (doi:10.1007/s00198-014-2857-4) contains supplementary material, which is available to authorized users.

\section{Keywords}

Bone Bisphosphonate Ex vivo micro-CT High-frequency loading Ovariectomized rats

\section{Introduction}

Osteoporosis is an emerging medical and socioeconomic threat characterized by a systemic impairment of bone mass and strength, which increases the propensity of fragility fractures [1]. From a patient's perspective, a fracture and the subsequent loss of mobility and autonomy often represent a major drop in quality of life. About $40 \%$ of postmenopausal women are affected by osteoporosis and, with an aging population, this number is expected to steadily increase in the near future $[\underline{2}, \underline{3}]$. Therefore, this disease has been an important public health issue because of the potentially devastating results and high prevalence in the population. Estrogen deficiency after menopause is one of the most common causes of osteoporosis in the population. In the estrogen-deficient state, the balance between bone resorption and bone formation shifts toward an increasing level of bone resorption, which results in the loss of bone mass and deterioration of the bone micro-architecture, since that adequate estrogen level is required to inhibit osteoclast maturation at cellular level and enhance the synthesis of cytokines that play roles in bone formation $[\underline{4}, \underline{5}]$. Pharmaceutical treatment with antiresorptive drugs, anabolic agents, as well as selective estrogen modulators is commonly used for postmenopausal osteoporosis []]. Although these drugs therapies are effective, most of them have some limitations and side effects that affect long-term administration and patient's adherence [ $[7]$. Hence, there is a clear need for the development of novel agents that exhibit a low level of toxicity and side effects as new treatment options for osteoporosis [1]. In this context, the profound anabolic effects of mechanical signals on bone have prompted the development of diverse biomechanical approaches in the treatment of osteoporosis, for preventing bone loss, increasing bone quantity, and/or improving its quality [8]. In particular, the mechanical signals provided by high-frequency (HF) vibration loading have been shown to promote bone formation, increase bone strength, and even recover bone loss arising from disabling or osteoporotic conditions $[\underline{9}, \underline{10}]$. However, the effects of HF vibration on bone tissue vary with vibration conditions, study design, timing of intervention, and at different anatomic sites $[\underline{10}, \underline{11}]$. 
In an effort to further elucidate the single and combined effect of HF loading and bisphosphonate treatment on ovariectomy-induced osteoporotic bone and to explore their potential for the treatment of osteoporosis, the present study investigated the impact of the exposure of long bones to HF mechanical loading via whole body vibration (WBV) on the bone micro-architecture. An animal study, with rats characterized by either untreated or bisphosphonate-treated osteoporotic bone, was conducted, with a twofold hypothesis: (i) HF loading via WBV improves the trabecular and cortical micro-architecture of ovariectomyinduced impaired bone and (ii) the concomitant application of mechanical treatment by means of HF WBV and pharmacological treatment by means of the antiresorptive drug alendronate results in a synergistic anabolic effect on the micro-architecture of osteoporotic bone.

\section{Materials and methods}

\section{Animals and experimental design}

A total of 84 female Wistar rats at 12 weeks of age were used in the present study. Fifty-five animals underwent ovariectomy surgery [OVX], while the remaining 29 animals were subjected to sham-ovariectomy surgery [shOVX]. (Sham)-ovariectomy surgery was performed at Charles River Laboratories (Charles River, L'Arbresle, France). For the rats subjected to sham surgery, the bilateral ovaries were lifted up and returned to their original position, while for the ovariectomized rats, the ovaries were removed. Rats arrived 5 days post-(sham)OVX surgery, with a body weight ranging between 220 and 250 g. Pair-feeding regimen was initiated immediately in an attempt to control the body weight gain throughout the study. The average daily food consumption of the shOVX animals was determined, and the quantified amount was then provided to the other animals. Animals were weighed at the start and once a week during the study. The OVX group was divided into 2 groups, an untreated group [OVX] $(n=26)$ and a group treated with the antiresorptive bisphosphonate drug alendronate [ALN] $(n=29)$. Alendronate sodium trihydrate (A4978-100MG, SigmaAldrich, Bornem, Belgium) was injected subcutaneously 3 days/week at a dose of $2 \mathrm{mg} / \mathrm{kg}$ body weight/dose, starting 5 days post-OVX surgery. Maintenance of bone tissue quantity and quality in OVX rats in response to such ALN dosage has been evidenced $[\underline{12}, \underline{13}]$. Saline administration $(0.9 \% \mathrm{NaCl})$ was performed according to the same time schedule to the rats of the OVX and shOVX groups. Injections were administered till the day of euthanasia. Each group (shOVX versus OVX versus ALN) was further divided into subgroups relative to the loading condition (sham-WBV versus WBV) and to the duration of the experimental period (4 days versus 14 days), resulting in 6 experimental groups for each time point (Cf. Table S1 Supplementary Material). The protocol of the animal experiment was approved by the ethical committee of KU Leuven (P050/2011), complied with ARRIVE guidelines for preclinical studies, and was performed according to the Belgian animal welfare regulations and guidelines.

\section{Vibration device and loading protocol}

HF mechanical loading was initiated 6 weeks post-(sham)ovariectomy. This time lapse was considered to be adequate for inducing significant bone changes in the rat long bones in response to ovariectomy [ $\underline{10}$ ]. The loading was applied by means of WBV via a custom-made vibration device, which was developed in collaboration with the Department of Mechanical Engineering, Division of Biomechanics KU Leuven (Cf. Fig. S1 Supplementary Material for detailed description of the vibration system) $[\underline{14}, 15]$. As stated above, the animals were randomly divided into 2 groups according to the duration of experimental period. In one 
group ( $n=43)$, the experiment lasted 4 days, while in the other group $(n=41)$, the experiment endured for 14 days. These time points were defined, based on the bone-anabolic potential of short-term application of HF loading in a bone-biomaterial healing context $[14, \underline{15}]$ and on the accelerated turnover of bone tissue after estrogen withdrawal, hypothesizing that the short-run application of HF loading on bone of OVX rats may be discernable at tissue micro-level. Each group was subdivided into two groups, a sham-WBV-unloaded (shWBV) and a WBV-loaded group. The WBV loading was applied for $10 \mathrm{~min} /$ day according to a protocol that consisted of 10 consecutive frequency steps $(130,135,140,145,150,130,135,140,145$, and $150 \mathrm{~Hz})$, each of these applied for $1 \mathrm{~min}$ at an acceleration of $0.3 \mathrm{~g}$. Frequency signals higher than those used in previous studies (superior to $90 \mathrm{~Hz}$ ) [1일 $\underline{16}-\underline{18}]$ were applied in the attempt to obtain an osteogenic effect of HF WBV for both trabecular and cortical bone, as suggested by Rubinacci and co-workers [19]. In addition, such loading frequencies demonstrated an anabolic effect on bone healing during implant osseointegration $[\underline{14}, \underline{15}]$. WBV was applied to the animals individually, taking into account the animal's body weight. Intervals of $24 \mathrm{~h}$ between the loading sessions were respected.

\section{Specimen preparation and micro-X-ray computed tomography analysis}

After euthanasia of the animals by cervical displacement under isoflurane-induced anesthesia, the hindlimbs were excised and the surrounding soft tissues removed. The tibiae were retrieved and immediately fixated in $10 \% \mathrm{CaCO}_{3}$-buffered formalin solution (pH 7.4) at $4{ }^{\circ} \mathrm{C}$ for $48 \mathrm{~h}$. The samples were further kept in the $70 \%$ ethanol at $4{ }^{\circ} \mathrm{C}$ until the day of micro-Xray computed tomography $(\mu \mathrm{CT})$ scanning.

For assessment of the bone micro-architectural changes in response to the hormonal status, mechanical loading status, and duration of the experimental period, the proximal part of the tibiae was examined ex vivo using a desktop $\mu \mathrm{CT}$ system, commercially available as Skyscan 1172 (Skyscan, Aartselaar, Belgium). During scanning, the tibia was placed in the polyethylene tube and immobilized inside the tubes by means of soft modeling clay. The bone samples were scanned along the midsagittal planes in the metaphyseal and mid-diaphyseal regions to obtain the $\mu \mathrm{CT}$ images. The scanning parameters were $6-\mu \mathrm{m}$ pixel size, $50-\mathrm{kV} \mathrm{X}$ ray voltage, $200-\mu \mathrm{A}$ electric current, and $0.5-\mathrm{mm} \mathrm{Al}$ filter. The scanning resulted in reconstructed 3D data sets with a voxel size of $6 \mu \mathrm{m}$, which were subsequently quantified using CTAn automated image analysis system (SkyScan, Aartselaar, Belgium). The registered gray-value images were segmented into binary images using a Gaussian filter and a fixed threshold (lower and the upper gray threshold values of 78 and 224, respectively) to extract the mineralized bone phase. Trabecular and cortical volumes of interest were defined, and the histomorphometric parameters were calculated according to the methodology described by Bouxsein and co-workers [20].

To determine the trabecular volume of interest (VOI) in the axial direction, the growth plate was determined as reference. The region of interest for the trabecular analysis started at the position of $1.5 \mathrm{~mm}$ downwards from the growth plate level and extended into the diaphysis over a distance of $3.3 \mathrm{~mm}$ (560 slices) (Fig. 1a). For each transverse slice, the VOI was delineated manually matching with the area occupied by trabecular bone (by freehand drawing — see Fig. 1b, c). The bone volume fraction (BV/TV), trabecular thickness (Tb.Th), trabecular separation (Tb.Sp), and trabecular number (Tb.N) were calculated 3D as measurements of trabecular bone mass and its distribution. The trabecular architecture was quantified by calculating the connectivity of the trabecular network (trabecular bone pattern formation, Tb.Pf) and the structure model index (SMI). Low Tb.Pf values reflect better connected trabecular lattices, whereas high Tb.Pf values indicate disconnected trabecular 
structures. The SMI values provide information regarding the trabecular shape, which is either rod- $(\mathrm{SMI}=3)$ or plate-like $(\mathrm{SMI}=0)$.
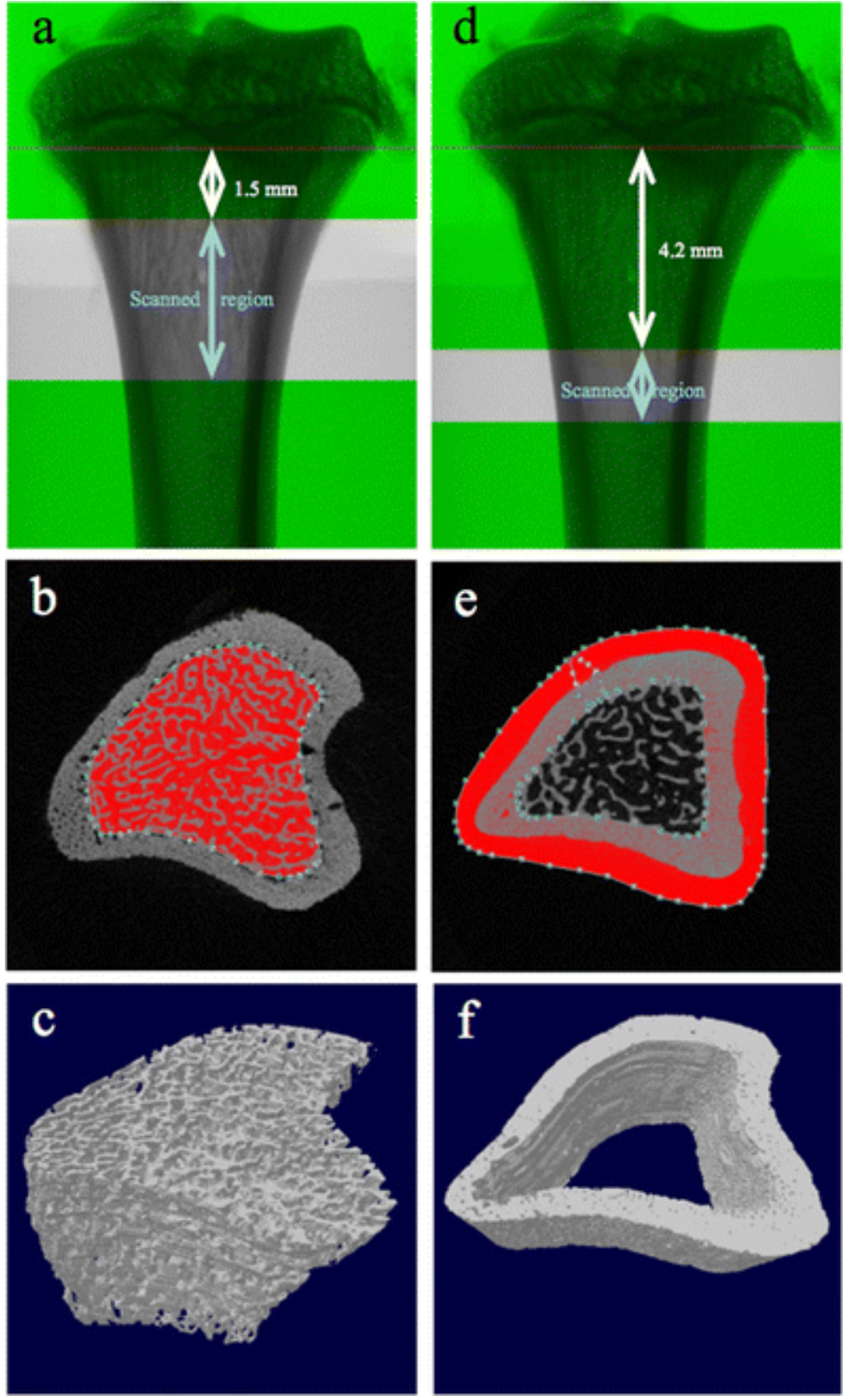

Fig. 1

Representative $\mu \mathrm{CT}$ images of tibia. a Region of interest (ROI) for trabecular bone analysis: $1.5 \mathrm{~mm}$ downwards from the growth plate level and extending into the diaphysis over a distance of $3.3 \mathrm{~mm}$. b Manual delineating of the volume of interest (red-highlighted region) of the trabecular bone, by freehand drawing of a line parallel and close to the endocortical boundary, thereby separating the trabecular from cortical bone. c 3D view of the trabecular bone volume reconstructed from the selected ROIs. d ROI for cortical bone analysis: $4.2 \mathrm{~mm}$ distally from the growth plate level and extending toward the distal diaphysis for $1.5 \mathrm{~mm}$. e Manual delineating of the volume of interest (VOI) of the cortical bone (gray-colored region), by freehand drawing starting at the endosteal side, continuing outside the cortical bone and ending with the closing of the loop. $f 3 D$ view of the cortical bone volume reconstructed from the selected ROI. More information on the method for ex vivo microCT analysis of rat bone can be found at http://umanitoba.ca/faculties/medicine/units/cacs/sam/media/ MN003 Bone microCT analysis rat.pdf 
To determine the cortical volume of interest in the axial direction, the growth plate was again used as reference. The region of interest of the cortical analysis was set with its closest edge at $4.2 \mathrm{~mm}$ distally from the growth plate and extended toward the distal diaphysis for $1.5 \mathrm{~mm}$ (250 slices) (Fig. 1d). For each transverse slice, the VOI was delineated manually (freehand drawing of cortical bone area—see Fig. 1e, f). The periosteal perimeter (Ps.Pm), medullary area (Ma.Ar), endocortical perimeter (Ec.Pm), mean polar moment of inertia (MMI), and eccentricity (Ecc) were calculated 2D as measurements of cortical bone mass and its distribution. The porosity (Ct.Po) and cortical thickness (Ct.Th) were calculated 3D as measurements of cortical bone mass and its distribution.

\section{Statistical analysis}

Results were expressed as means and confidence intervals. The data were analyzed by threeway factorial analysis of variance (ANOVA) and pairwise comparison tests in order to assess the effect of hormonal status, mechanical status, duration of the experimental period (i.e., the three independent variables), and their interactions on the trabecular and cortical bone microarchitectural changes. Post hoc tests for pairwise comparisons were performed using the Tukey HSD test with Bonferroni correction. The statistical software package SPSS (SPSS ver. 13.0, Chicago, IL, USA) was used. Differences were considered significant at $p<0.05$.

\section{Results}

\section{The body weight of OVX rats increased despite of the pair-feeding regime and HF loading}

The body weight recordings at the day of arrival of the animals (i.e., 5 days post-(sh)OVX surgery) revealed a slightly higher body weight for OVX animals compared to shOVX animals (235.6 versus 224.5 g, respectively). As a consequence of ovariectomy surgery, OVX animals indeed have the tendency to gain weight. However, 6 weeks later, it was observed that the established pair-feeding regimen was able to control the body weight gain in OVX animals (14.5 versus $14.5 \mathrm{~g}$ for OVX and shOVX animals, respectively). Surprisingly, at week 8 post-(sh)OVX surgery (corresponding to the end of the 14 days of WBV intervention), an almost twofold body weight gain of the OVX rats compared to that of shOVX animals was recorded (37.8 versus $14.05 \mathrm{~g}$, respectively), despite the uninterrupted pair-feeding regime. Furthermore, independently of the allocated experimental group, ALN animals displayed a three- and twofold weight increase compared to the weight gain of shOVX and OVX, respectively. Finally, HF WBV application did not affect the rats' body weight gain in all experimental groups (Cf. Fig. S2 Supplementary Material).

\section{ANOVA revealed significant effect of hormonal status, mechanical loading, duration of the experimental period, and their interactions on cortical and trabecular micro-structural parameters}

For the trabecular bone structural parameters, a three-way interaction between the hormonal status, the mechanical loading status, and the duration of the experimental period was found, implying that subsequent statistical analyses should make use of the full factorial model (Tables $\underline{1}$ ). Within this model, pairwise comparisons were performed with Bonferroni corrections up to a significance level of $p<0.00417$ for the hormonal status and $p<0.0083$ for the mechanical status and for the duration of experimental period. The results are graphically presented in Fig. $\underline{2}$ and are discussed in detail below. For the cortical bone 
analysis, only two-way interactions between the independent variables were found (Table 2). Hence, a customized factorial model was used, and subsequent pairwise comparisons were performed with Bonferroni corrections at significance level of $p<0.0167$ for the hormonal status and $p<0.0083$ for the mechanical status and for the duration of experimental period. As two two-way interactions displayed significant results for the cortical bone (hormonal*mechanical and hormonal*duration), the results were displayed in separate figures. In Fig. $\underline{3}$, the impact of the hormonal status on the cortical bone parameters, relative to the loading condition, is displayed (data of the 4 days and 14 days experiment are pooled).

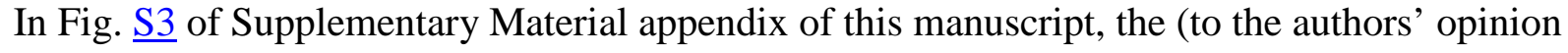
less relevant) results of the impact of the hormonal status relative to the duration of the experimental period on the cortical bone parameters are presented.

\section{Table 1}

ANOVA results of the effect of the independent variables (i.e., the hormonal status, the mechanical status, the duration of the experimental period, and their interactions) on the trabecular bonedependent variables (i.e., BV/TV, Tb.Th, Tb.Sp, Tb.N, Tb.Pf, SMI)

\begin{tabular}{|c|c|c|c|c|c|c|c|}
\hline & 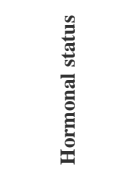 & 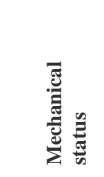 & 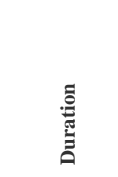 & 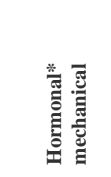 & 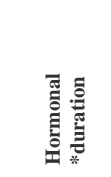 & 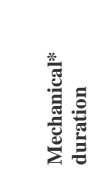 & 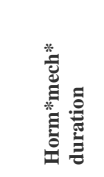 \\
\hline $\mathrm{BV} / \mathrm{TV}$ & $p<0.001 *$ & $p=0.608$ & $p=0.379$ & $p=0.095$ & $p=0.064$ & $p=0.360$ & $p=0.042^{*}$ \\
\hline Tb.Th & $p=0.003^{*}$ & $p=0.840$ & $p<0.001^{*}$ & $p=0.243$ & $p<0.001 *$ & $p=0.505$ & $p=1.000$ \\
\hline Tb.Sp & $p<0.001^{*}$ & $p=0.054$ & $p=0.018^{*}$ & $p=0.030^{*}$ & $p=0.003^{*}$ & $p=0.602$ & $p=0.676$ \\
\hline Tb.N & $p<0.001^{*}$ & $p=0.516$ & $p=0.089$ & $p=0.303$ & $p=0.482$ & $p=0.348$ & $p=0.087$ \\
\hline Tb.Pf & $p<0.001^{*}$ & $p=0.939$ & $p=0.002^{*}$ & $p=0.629$ & $p=0.010^{*}$ & $p=0.557$ & $p=0.677$ \\
\hline
\end{tabular}




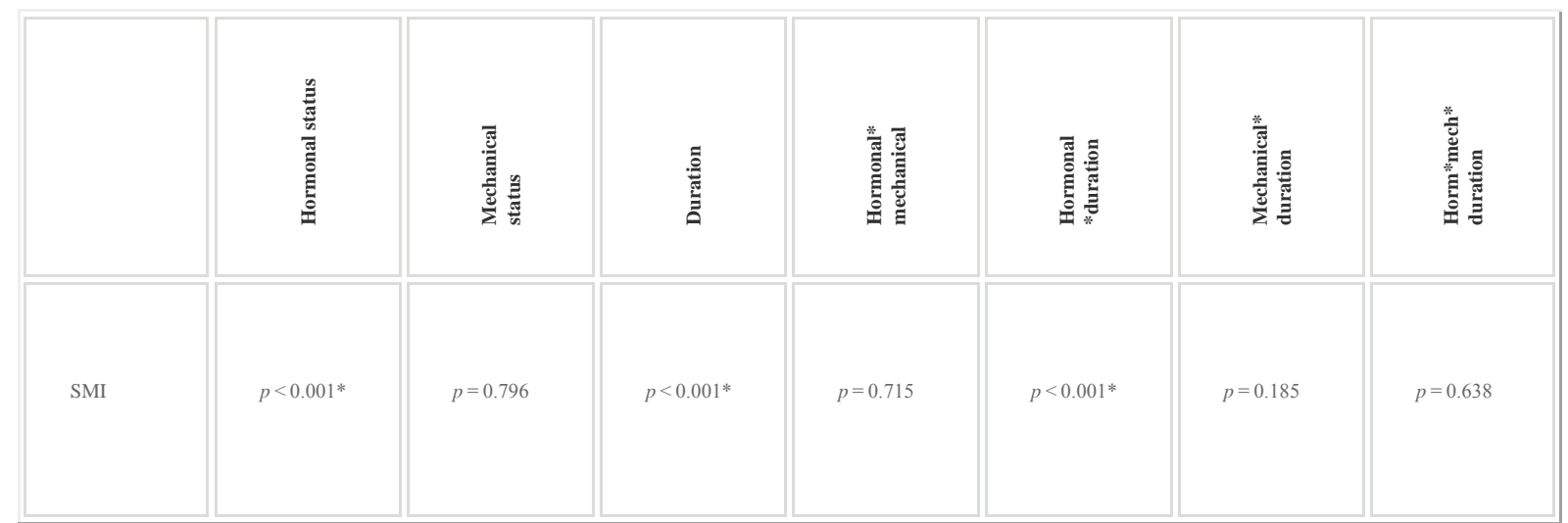

BV/TV bone volume/tissue volume, $T b . T h$ trabecular thickness, $T b . S p$ trabecular spacing, $T b . N$ trabecular number, $T b . P f$ trabecular bone pattern formation, SMI structure model index; ${ }^{\star} p<0.05$

$$
\text { 4days: } \square \text { shovX } \triangle \text { OVX OALN }
$$
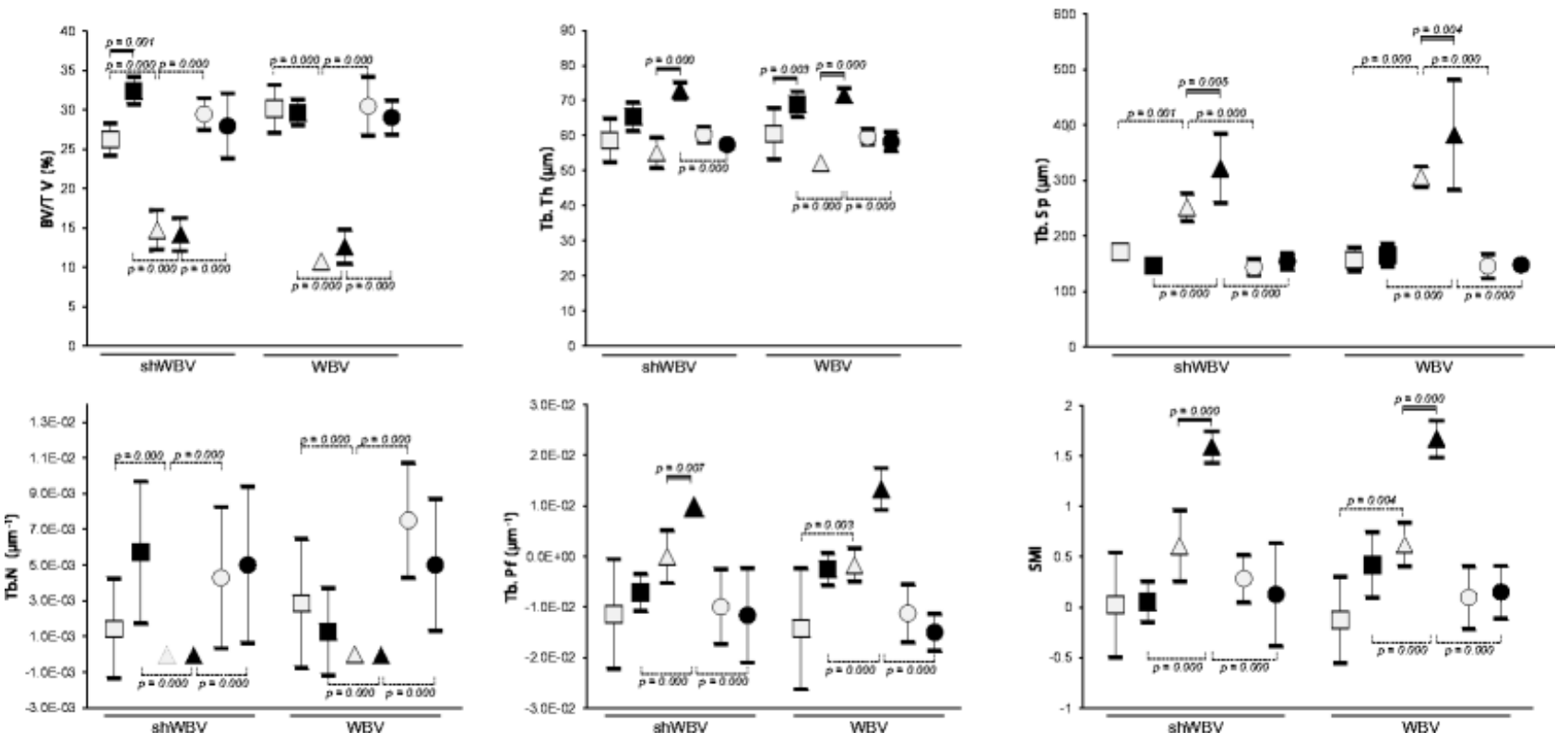

Fig. 2

Results of micro-CT analysis of the trabecular bone structural parameters according to the animal's hormonal status, the mechanical loading status, and the duration of the experimental period, for bone volume/tissue volume (BV/TV, \%); trabecular thickness (Tb.Th, $\mu \mathrm{m}$ ); trabecular separation (Tb.Sp, $\mu \mathrm{m}$ ); trabecular number (Tb.N, $\mu \mathrm{m}^{-1}$ ); trabecular bone pattern formation (Tb.Pf, $\mu \mathrm{m}^{-1}$ ); and structural model index (SMI). Dotted connection bars denote statistically significant results of the pairwise comparisons between the groups with differing hormonal status (shOVX versus OVX versus ALN). Double line connection bars display the statistically significant differences between groups with differing experimental duration (4 versus 14 days). Open versus filled symbols refer to 4 and 14 days data, respectively

\section{Table 2}

ANOVA results of the effect of the independent variables (i.e., the hormonal status, the mechanical status, the duration of the experimental period, and their interactions) on the cortical bone-dependent variables (i.e., Ct.Po, Ps.Pm, Ma.Ar, Ec.Pm, MMI, Ecc, Ct.Th)

\begin{tabular}{|c|l|l|l|l|l|}
\hline & $\begin{array}{l}\text { Hormonal } \\
\text { status }\end{array}$ & $\begin{array}{l}\text { Mechanical } \\
\text { status }\end{array}$ & Duration & Hormonal*mechanical & Hormonal*duration \\
\hline \hline Ct.Po & $p=0.002 *$ & $p=0.800$ & $p=0.000^{*}$ & $p=0.821$ & $p=0.000^{*}$ \\
\hline
\end{tabular}




\begin{tabular}{|c|c|c|c|c|c|}
\hline & $\begin{array}{l}\text { Hormonal } \\
\text { status }\end{array}$ & $\begin{array}{l}\text { Mechanical } \\
\text { status }\end{array}$ & Duration & Hormonal*mechanical & Hormonal*duration \\
\hline Ps.Pm & $p=0.000 *$ & $p=0.013 *$ & $p=0.047^{*}$ & $p=0.026^{*}$ & $p=0.772$ \\
\hline Ma.Ar & $p=0.000 *$ & $p=0.001 *$ & $p=0.178$ & $p=0.057$ & $p=0.230$ \\
\hline Ec.Pm & $p=0.000 *$ & $p=0.058$ & $p=0.000^{*}$ & $p=0.256$ & $p=0.000^{*}$ \\
\hline MMI & $p=0.000 *$ & $p=0.127$ & $p=0.904$ & $p=0.027^{*}$ & $p=0.496$ \\
\hline Ecc & $p=0.115$ & $p=0.258$ & $p=0.375$ & $p=0.988$ & $p=0.943$ \\
\hline Ct.Th & $p=0.000 *$ & $p=0.002 *$ & $p=0.000^{*}$ & $p=0.671$ & $p=0.030^{*}$ \\
\hline
\end{tabular}

Ct.Po cortical porosity, Ps.Pm periosteal perimeter, Ma.Ar medullary area, Ec.Pm endocortical perimeter, MMI mean polar moment of inertia, Ecc eccentricity, Ct.Th cortical thickness; ${ }^{\star} p<0.05$

$\square$ shovx $\triangle$ OVX OALN
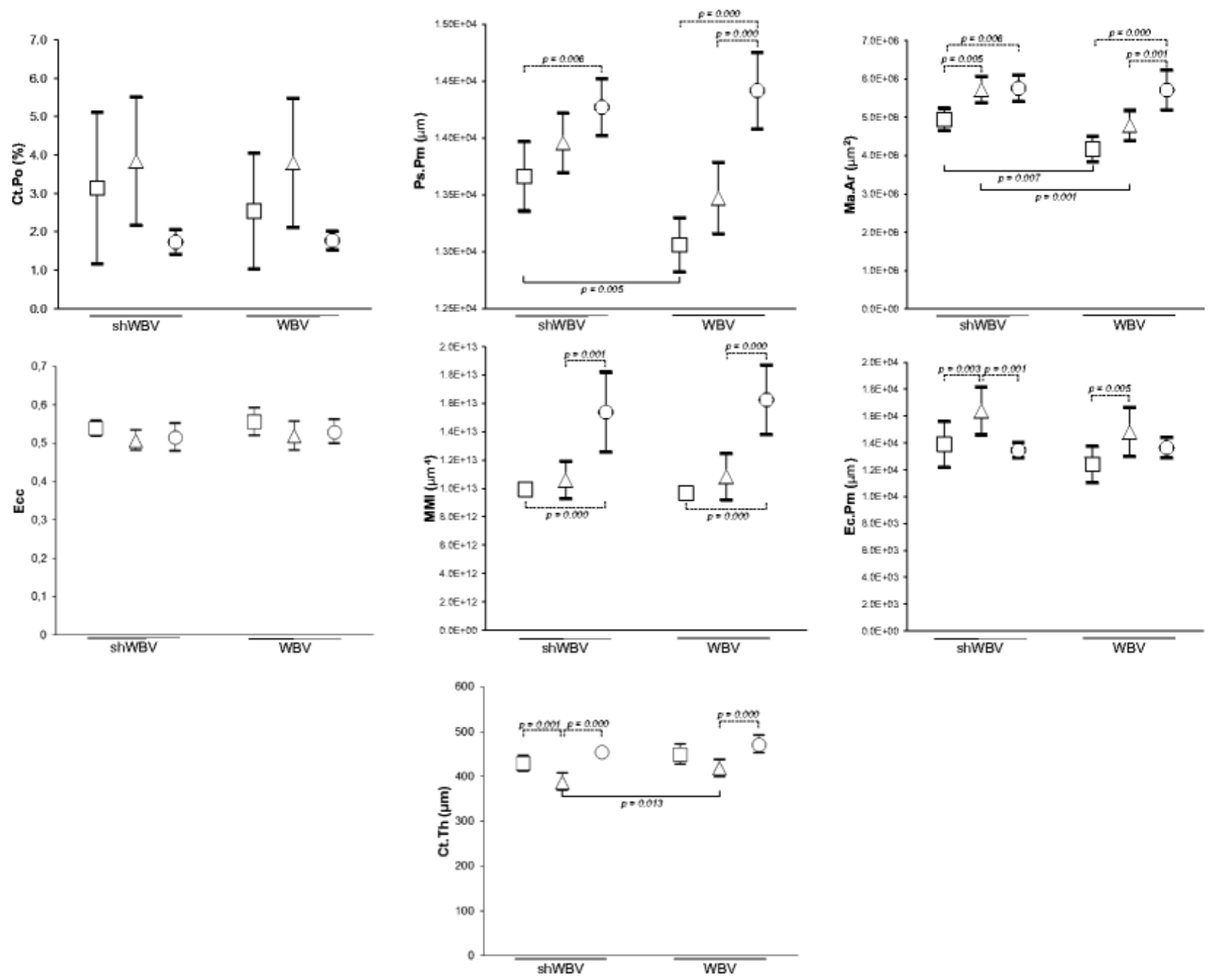

Fig. 3

Results of micro-CT analysis of the cortical bone structural parameters according to the animal's hormonal condition and the mechanical loading status, for cortical porosity (Ct.Po, \%); periosteal 
perimeter (Ps.Pm, $\mu \mathrm{m})$; endocortical perimeter (Ec.Pm, $\mu \mathrm{m})$; cortical thickness (Ct.Th, $\mu \mathrm{m})$; medullary area (Ma.Ar, $\left.\mu \mathrm{m}^{2}\right)$; mean polar moment of inertia (MMI, $\mu \mathrm{m}^{4}$ ); and eccentricity (Ecc). Owing to the lack of a 3-way interaction, the data of the 4 days and 14 days groups were pooled when analyzing the impact of the hormonal status on the cortical bone parameters, relative to the loading condition. Dotted connection bars denote statistically significant results of the pairwise comparisons between the groups with differing hormonal status (shOVX versus OVX versus ALN). Plain connection bars display the statistically significant differences between groups with differing mechanical status (shWBV versus WBV). The reader is referred to Fig. S1 of Supplementary Material for the results of the impact of the hormonal status relative to the duration of the experimental period on the cortical bone parameters

\section{Results of the trabecular bone structural parameters}

\section{(i)}

Trabecular bone micro-architecture was affected mainly by hormonal status.

The trabecular bone of OVX animals displayed a significantly decreased BV/TV and Tb.N, and a significant increased Tb.Sp, Tb.Pf, and SMI, compared to shOVX or ALN animals, evidencing the status of ovariectomy-induced osteopenia in the OVX group (Fig. 2). No statistically significant differences for all trabecular parameters were found between ALN and shOVX groups, thereby providing evidence for the efficacy of pharmacology treatment with alendronate in preventing ovariectomy-induced trabecular bone micro-architectural changes (Fig. 2).

(ii)

The applied HF loading did not affect the trabecular bone micro-architecture.

Unlike the major impact of the hormonal status on the trabecular bone structural parameters, the mechanical intervention with HF stimuli did not affect the trabecular micro-architecture.

(iii)

Trabecular bone micro-architectural changes within a 10-day time span can be observed by $\mu \mathrm{CT}$.

Temporal alterations of all parameters, except of Tb.N, could be captured over a 10-day period (from 4 days till 14 days) (Fig. 2). In particular, the OVX group was prone to shortterm bone trabeculae evolutions with thickening of the bone trabeculae, increased spacing and a more rod-like shape, and more disconnected trabecular structures.

\section{Results of the cortical bone structural parameters}

\section{(i)}

Also the cortical bone micro-architectural parameters are primarily affected by the hormonal status, in particular by ALN.

The animals' hormonal status exerted an effect on the cortical bone micro-architecture, independently of the duration of experimental period. OVX group in comparison to ALN showed a statistically significant decrease in Ps.Pm (OVX-WBV versus ALN-WBV), Ma.Ar (OVX-WBV versus ALN-WBV), MMI (OVX versus ALN), Ct.Th (OVX versus ALN), and an increase in Ec.Pm (OVX-shWBV versus ALN-shWBV) (Fig. ㄱ) ). When compared to the shOVX group, OVX presented a thinner cortical thickness (OVX-shWBV versus shOVXshWBV) and an increased Ec.Pm (OVX versus shOVX) and Ma.Ar (OVX-shWBV versus shOVX-shWBV). In contrast to the trabecular bone results, comparisons between ALN and shOVX groups showed statistically significant differences for Ps.Pm, Ma.Ar, and MMI (Fig. 3 ).

(ii)

HF loading affected the cortical micro-architecture, though not synergistically with ALN.

HF WBV mechanical treatment positively affected the shOVX cortical bone by decreasing Ma.Ar, and the OVX group by decreasing Ma.Ar accompanied by an increase in Ct.Th. 
Furthermore, a negative effect of WBV was observed in shOVX group, with a reduction in Ps.Pm. Finally, the mechanical loading did not result in any significant effect in the ALN group (Fig. $\underline{3}$ ).

\section{Discussion}

In the present study, it was evaluated how mechanical stimulation by means of HF loading of ovariectomized-induced osteoporotic bone, combined or not with pharmacological treatment (bisphosphonates), can affect the bone's micro-structure. Hence, high-resolution microcomputed tomography $(\mu \mathrm{CT})$ was used to determine the changes in bone micro-architecture, since this method enables the quantitative assessment of the skeleton's morphology thereby providing identification of patients with osteoporosis as well as evaluation of the impact of treatments [21]. It was hypothesized that (i) HF WBV loading improves the microarchitecture of ovariectomy-induced impaired bone and that (ii) the concomitant application of HF WBV and bisphosphonates results in a synergistic anabolic effect on the bone microarchitecture. The main findings of this study are represented schematically in Fig. $\underline{4}$, with the trabecular bone drawn as the inner circle with grids pattern and the cortical bone the gray halo surrounding the inner circle. The following findings can be highlighted: (i) HF loading via WBV did not affect trabecular bone but stimulated cortical bone, enhancing its thickness in OVX rats; (ii) although ALN was able to prevent ovariectomy-induced bone loss, its concomitant application with HF loading did not produce a synergistic bone response; and (iii) the hormonal status, characterized by the presence of estrogen in shOVX groups and the lack of this hormone in OVX group, displayed the major impact on the trabecular and cortical bone micro-structural parameters evaluated. 
a - MECHANICAL STATUS
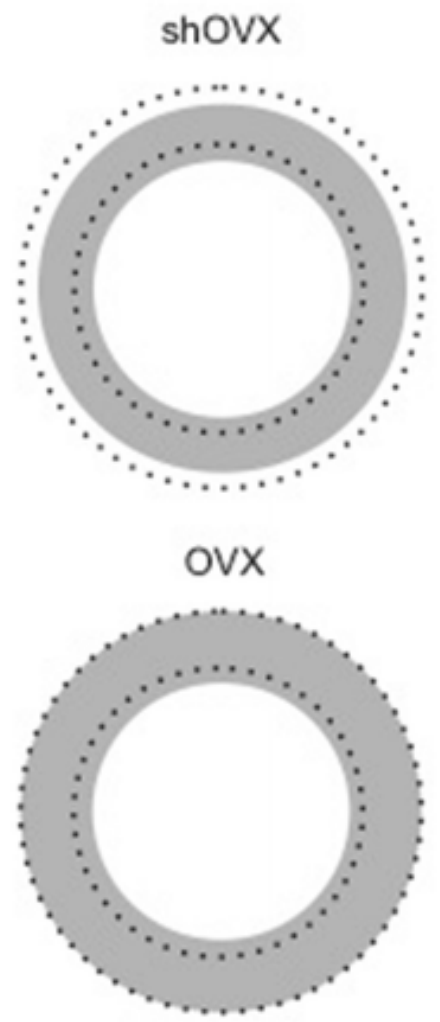

ALN

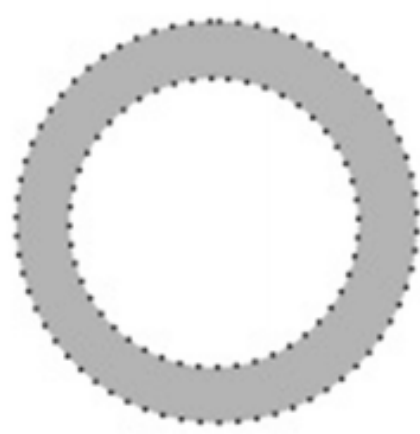

\section{b - HORMONAL STATUS}

OVX vs shOVX
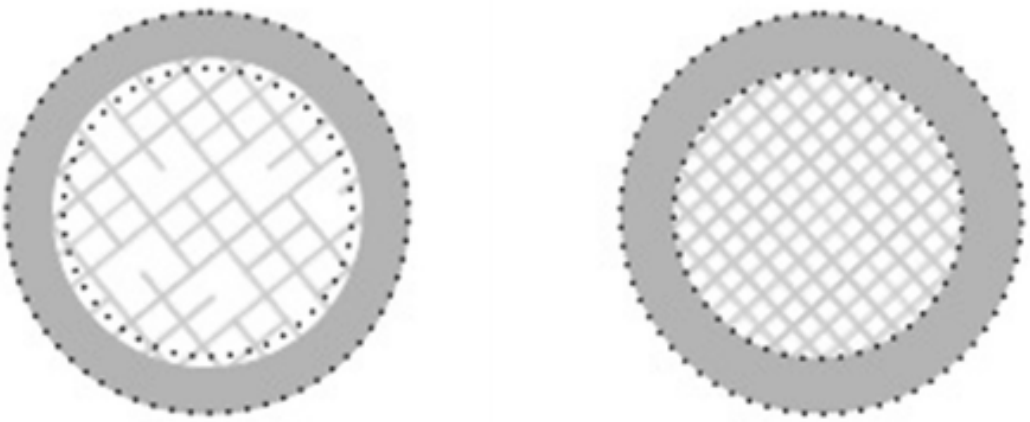

\section{OVX vs ALN}
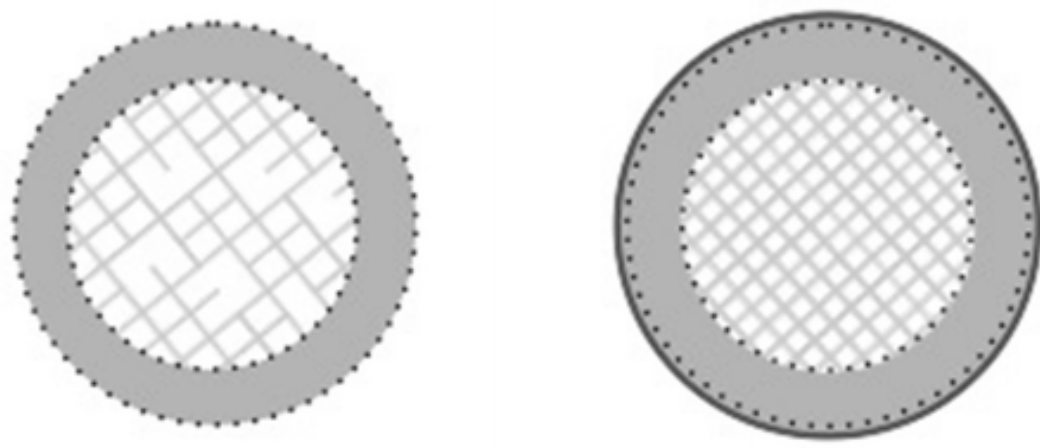

shOVX vs ALN
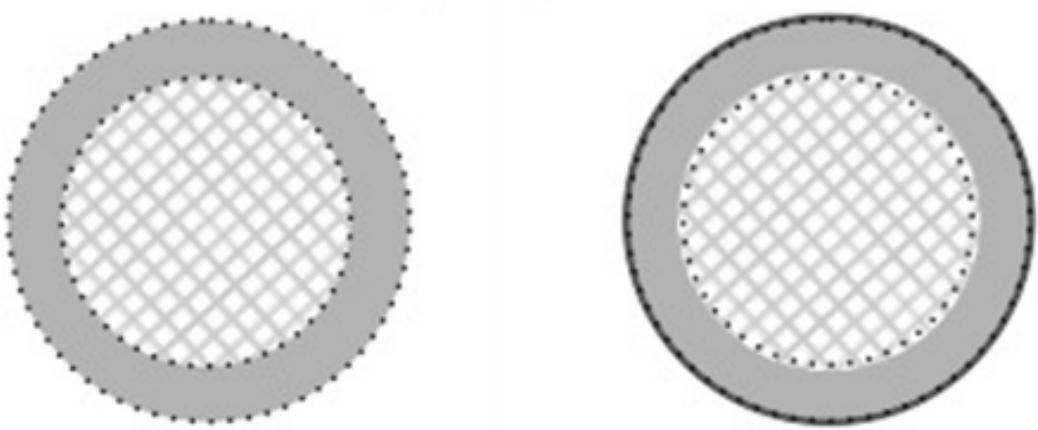

Fig. 4

Schematically, representation of the main results of the bone morphometric parameters changes in response to mechanical and/or pharmaceutical treatment. The results for the parameters SMI, MMI, Ec.Pm, and Ecc are not included in the figure, as the schematic presentation of these parameters is not possible. Trabecular bone is represented by the inner circle with grids pattern and cortical bone by the gray halo surrounding the inner circle. a Impact of mechanical status on bone micro-architecture. HF loading via WBV did not affect trabecular bone (trabecular bone not shown) but affected cortical bone. The resulting effect of HF WBV on the cortical bone is represented by the gray halo, while the dotted circumferences represent the initial condition (i.e., prior to mechanical stimulation; shWBV). After application of HF WBV for an extended period of time, the shOVX group showed a reduction in Ps.Pm and Ma.Ar. The OVX group presented a decrease in Ma.Ar accompanied by an increase in Ct.Th. No changes in response to HF WBV were observed in the cortical bone structural parameters of the ALN group. $\mathbf{b}$ Impact of hormonal status on bone micro-architecture. In trabecular bone, OVX animals displayed a significant decreased in BV/TV and Tb.N, and a significant increased Tb.Sp and Tb.Pf when compared to shOVX and ALN animals. Furthermore, no statistically significant differences for all trabecular parameters were noted between ALN and shOVX groups. In cortical bone, OVX in 
comparison to shOVX presented an increase in Ma.Ar and Ec.Pm and consequently a thinner cortical thickness. Comparisons between OVX and ALN group showed an increase in Ps.Pm and in Ct.Th in ALN group. In contrast to the trabecular bone results, comparisons between ALN and shOVX groups showed statistically significant differences, in particular an increased Ps.Pm and Ma.Ar. The plain gray halo represents the bone structural changes resulting from the hormonal status; the dotted line circumferences the reference bone condition. A bold outline of the cortical bone indicates an increased periosteal perimeter

Indeed, estrogen is the major hormonal regulator of bone metabolism in women [22]. The ovariectomized rat model was used to simulate postmenopausal osteoporosis [23], and the resulting withdrawal of estrogen after ovariectomy surgery induces a rapid increase in bone turnover associated with a substantial bone loss [22]. As a consequence of this unbalanced process, a compromised bone condition was established in the OVX group, as quantified in the present study by the reduction of BV/TV, Tb.N, Tb.Th, and Ct.Th and by an increase in SMI, Tb.Pf, Tb.Sp, Ma.Ar, and Ec.Pm as compared to shOVX group. Similar changes in bone structural parameters after ovariectomy have been reported in other reports [16, 17, 24, 25]. Nonetheless, the pharmacology treatment of OVX rats with the bisphosphonate drug alendronate was able to prevent ovariectomy-induced bone loss. Our results consistently indicated the preservation of the trabecular and the cortical bone in response to ALN administration in OVX rats, corroborating with the findings from clinical studies reporting a favorable effect of ALN treatment on the bone in postmenopausal women [26]. ALN is an antiresorptive agent preventing osteoporosis-related deterioration of bone by decreasing the bone resorption [27]. More specifically, ALN is a nitrogen-containing bisphosphonate, which inhibits the enzyme farnesyl pyrophosphate (FPP) synthase, thereby disrupting the production of isoprenoid lipids in the mevalonate pathway, preventing the prenylation of small GTPase proteins necessary for osteoclast function and causing accumulation of a toxic, isoprenoidcontaining metabolite that disrupts osteoclast function and survival [28]. As a result of the bone resorption suppression by ALN administration, trabecular bone structural parameters were maintained in ovariectomized rats, with similar values as those of the shOVX rats (normal bone). In the cortical bone, besides preserving the bone structural parameters affected by ovariectomy, ALN bone compared to shOVX bone displayed decreased (though not significantly) cortical porosity (Ct.Po) and significantly increased periosteal perimeter (Ps.Pm), mean polar of inertia (MMI), and medullar area (Ma.Ar). In agreement with these results, previous studies have reported that bisphosphonates reduce remodeling and therefore decrease cortical porosity by increasing the uniformity of mineralization [29]. Also, periosteal expansion occurs in response to ALN treatment, thereby increasing the medullary area and cortical thickness compared to control bone [30]. Consequently, these cortical bone structural changes might contribute for improving the bone biomechanical behavior, which could reduce the incidence of bone fractures in patients with osteoporosis.

Unlike pharmacological treatment with ALN, the mechanical treatment with HF via WBV did not improve the trabecular bone structural parameters in OVX group. The lack of success of the HF loading treatment on the trabecular bone can potentially be associated to its short-term exposure as well as to the withdrawal of estrogen after ovariectomy in this group. Estrogen appears to possess a permissive role on the osteogenic effects of mechanical loading on bone through the involvement of $\alpha$-form estrogen receptor (ER- $\alpha$ ) in the mechanosensing pathway of bone cells [31-33]. As ER- $\alpha$ expression in osteoblasts and osteocytes depends on estrogen concentration [34], a failure to maintain bone mass and strength after the menopause might be due to a reduction in the activity or number of ER- $\alpha$ in bone cells, thereby limiting their anabolic response to mechanical loading and allowing bone loss comparable to that associated with disuse [31]. In contrast to these trabecular bone findings, an osteogenic effect of HF mechanical loading was observed in the cortical bone of OVX group, resulting in a significant smaller medullar area and thicker cortical thickness. In agreement with these results, 
Rubinacci and co-workers reported that the osteogenic potential of WBV is limited to the bone cortex in OVX rats [19]. Such results might be a consequence of the stimulation of the periosteal bone by mechanical loading after the menopause [30]. Increased periosteal apposition causes the cortical shell to move further from the long axis of bone, and the larger bone size improves their resistance to fractures [35]. This implies that it is possible to lose total bone mass but still have equally or elevated mechanically competent bones since that increases in the cortical thickness will increase the bone bending moment and consequently its strength, enhancing its overall mechanical competence [35].

Cortical bone remodeling is a direct consequence of the surface-specific effects of estrogen on bone in response to loading. However, it is important to note that besides ER- $\alpha$, estrogen has also a $\beta$-form of receptor involved in the mechanotransduction. These receptors have opposing effects on bone formation, and their expression differs on each bone surface as well as during the different stages of osteoblast differentiation [36]. Signaling through ER- $\alpha$ enhances mechanically induced bone formation $[\underline{31}, 32]$ while signaling through ER- $\beta$ suppresses mechanically induced periosteal bone formation [36, 37]. Consequently, in a state of estrogen deficiency, as in the menopause, the lack of inhibitory ER- $\beta$ signaling might activate a compensatory mechanism in order to maintain bone mechanical resistance despite the loss of bone at the endocortical surface $[\underline{19}, \underline{36}]$. In contrast to the OVX group where a suppression of ER- $\beta$ signaling is suggested as potential mechanism of action, activation of this receptor by the estrogen presence might have occurred in shOVX group. This fact can explain why the mechanical loading treatment of shOVX induced a negative response at the cortical region, with a reduction in the periosteal perimeter. In line with this assumption, Saxon and co-workers demonstrated that the treatment with estrogen, even at low dose, suppressed periosteal bone formation occurring after mechanical loading [38]. The authors reported that the osteogenic loading response at the periosteal surface was reduced through estrogen treatment by $48 \%$ after 1 week of mechanical loading, and this decrease in bone formation caused reductions in the loading-induced gains in cortical area, moment of inertia, and bone mineral content after 5 weeks of loading [38].

Despite the cortical bone-anabolic response in OVX animals to the mechanical HF WBV intervention as well as to the pharmacological treatment with ALN, the concomitant application of both treatments did not produce a synergistic bone response. The values of the bone micro-structural parameters for ALN-shWBV and ALN-WBV groups were in the same range, suggesting the lack of effect of WBV in the ALN group. Previous studies showed similar results and attested that WBV exercise and ALN did not have any additive effects on the lumbar spine bone mineral density [39] and on the urinary and serum bone formation levels in postmenopausal women [40]. Likewise, Lespessailles and co-workers reported that zoledronic acid and running exercises did not produce any additive effects on bone mass and strength [41], whereas Fuchs et al. showed that combination of running exercise and ALN was more beneficial in preventing declines in bone mass and strength than either intervention alone [42]. Compared with the study of Fuchs et al., a higher dose of ALN was used in the current study $(0.015 \mathrm{mg} / \mathrm{kg}$ body weight at 2 days/week versus $2 \mathrm{mg} / \mathrm{kg}$ body weight at 3 days/week). Therefore, the effect of mechanical loading on the bone micro-architecture could possibly be masked by the strong influence of alendronate on bone in the present study. Furthermore, in the referred study, a different kind of mechanical stimulation was applied, in which the rats were exercised on a motorized treadmill 5 days/week at $5 \%$ incline for 14 weeks. Finally, HF WBV loading was applied in the present study for only a limited period of time. Further studies are therefore necessary and ongoing, for evaluating the interaction of different dosages of alendronate with WBV, applied for varying time periods, on the bone micro-architecture and strength. 
Similarly to the pharmacologic treatment with ALN, no association could be found between the mechanical status and the duration of the experimental period. Nonetheless, there was a significant association between the hormonal status and the duration of the experimental period, indicating that some bone parameter changes could be captured within a 10-day period. In particular, the trabecular bone (Tb.Th, Tb.Sp, Tb. Pf, SMI) of the OVX rat seemed to be prone to short-term changes. Such evolutions have been reported by others though with observation intervals of 4 weeks or more [ $\underline{43}, \underline{44}$. Noteworthy mentioning is that the increased body weight and resulting increased skeletal loading of the OVX animals was unable to remediate these bone deterioration changes. To avoid this body weight gain in OVX group, pair-feeding was established immediately after the rats' arrival but was apparently unable to control the weight gain during the entire experimental period. The effect of OVX on subsequent gain in fat tissue has been extensively examined and has been associated with more adipogenesis than osteogenesis [45].

A limitation of the present experimental set-up is the short period of mechanical HF intervention (4 or 14 days). It could be that longer periods of vibration therapy would have induced more pronounced effects on bone mass. However, in other studies using OVX rodents and applying WBV therapy for 8 weeks or more, a substantial effect of this mechanical loading scheme on bone was neither found $[\underline{17}, \underline{25}]$. Other factors like the study design and the loading frequency could play a more prominent role in the response of the bone tissue to WBV. Furthermore, the biomechanical properties of the bone were not evaluated in this study. As osteoporosis is characterized by decreased bone volume, microarchitectural deterioration, decreased bone strength, and increased fracture risk, future studies evaluating how the changes in bone micro-structure in response to different treatments (mechanical or pharmacological) contribute to bone biomechanical properties are necessary. Finally, whether the cortical bone response to HF loading was due to increased bone formation or decreased bone resorption could not be answered as no dynamic bone histomorphometry nor the kinetic evaluation of the bone response (via the inclusion of more time points) was applied.

Within the limitations of this study, it can be concluded that HF WBV mechanical stimulation can have an anabolic effect on the cortical bone part of ovariectomy-induced osteoporotic rats. However, the osteoporotic trabecular bone seemed to be less prone to HF WBV loading influences. Additionally, the association of this mechanical loading treatment with alendronate administration did not produce any additive effect on the bone micro-architectural response, when compared with ALN therapy alone, suggesting that the selected ALN dosage might have masked the effect of HF WBV on the bone. Hence, the study hypotheses could only partially be sustained.

Acknowledgments

The author would like to acknowledge Dr. A. Ivanova for the help with the statistical analysis. This work was supported by the Fund for Scientific Research Flanders (FWO Flanders—-postdoctoral researcher K. Vandamme) and by the Brazilian Science Without Borders Program (246131/2012-8 process, Phd student G.V. Camargos; 245450/2012-2 process, Postdoctoral researcher F. Faot). Conflicts of interest

The authors Kouki Hatori, Germana De Villa Camargos, Marissa Chatterjee, Fernanda Faot, Keiichi Sasaki, Joke Duyck, and Katleen Vandamme declare that there are no conflicts of interest related to the manuscript. 


\section{Electronic supplementary material}

Below is the link to the electronic supplementary material.

\section{ESM 1 (DOCX $4726 \mathrm{~kb}$ )}

References

1.

Rachner TD, Khosla S, Hofbauer LC (2011) Osteoporosis: now and the future. Lancet 377:1276-1287. doi:10.1016/S0140-6736(10)62349-5

2.

Melton LJ III, Chrischilles EA, Cooper C et al (2005) How many women have osteoporosis? J

Bone Miner Res 7:1005-1010

3.

Burge R, Dawson-Hughes B, Solomon DH et al (2007) Incidence and economic burden of osteoporosis-related fractures in the United States, 2005-2025. J Bone Miner Res Off J Am Soc Bone Miner Res 22:465-475

4.

Inada M, Miyaura C (2010) Cytokines in bone diseases. Cytokine and postmenopausal osteoporosis. Clin Calcium 20:1467-1472

5.

Pacifici R (1996) Estrogen, cytokines, and pathogenesis of postmenopausal osteoporosis. J Bone Miner Res Off J Am Soc Bone Miner Res 11:1043-1051

6.

Sambrook P, Cooper C (2006) Osteoporosis. Lancet 367:2010-2018

7.

Siris ES, Selby PL, Saag KG et al (2009) Impact of osteoporosis treatment adherence on fracture rates in North America and Europe. Am J Med 122:S3-S13

8.

E O, Luu Y, B A, et al. (2010) Mechanical signals as anabolic agents in bone. Nature 6:50-59 9.

Judex S, Rubin CT (2010) Is bone formation induced by high-frequency mechanical signals modulated by muscle activity? J Musculoskelet neuronal Interact 10:3-11

10.

Judex S, Lei X, Han D, Rubin C (2007) Low-magnitude mechanical signals that stimulate bone formation in the ovariectomized rat are dependent on the applied frequency but not on the strain magnitude. J Biomech 40:1333-1339

11. 
Oxlund B, Ørtoft G, Andreassen T, Oxlund H (2003) Low-intensity, high-frequency vibration appears to prevent the decrease in strength of the femur and tibia associated with ovariectomy of adult rats. Bone 32:69-77. doi:10.1016/S8756-3282(02)00916-X

12.

Chen B, Li Y, Yang X, Xie D (2013) Comparable effects of alendronate and strontium ranelate on femur in ovariectomized rats. Calcif Tissue Int 93:481-486

13.

Chen B, Li Y, Xie D, Yang X (2012) Low-magnitude high-frequency loading via whole body vibration enhances bone-implant osseointegration in ovariectomized rats. J Orthop Res 30:733-739. doi:10.1002/jor.22004

14.

Ogawa T, Possemiers T, Zhang X et al (2011) Influence of whole-body vibration time on peri-implant bone healing: a histomorphometrical animal study. J Clin Periodontol 38:180185. doi:10.1111/j.1600-051X.2010.01637.X

15.

Ogawa T, Zhang X, Naert I et al (2011) The effect of whole-body vibration on peri-implant bone healing in rats. Clin Oral Implants Res 22:302-307. doi:10.1111/j.1600-0501.2010. $\underline{02020 . x}$

16.

Tezval M, Biblis M, Sehmisch S et al (2011) Improvement of femoral bone quality after lowmagnitude, high-frequency mechanical stimulation in the ovariectomized rat as an osteopenia model. Calcif Tissue Int 88:33-40

17.

Brouwers JEM, Van Rietbergen B, Ito K, Huiskes R (2010) Effects of vibration treatment on tibial bone of ovariectomized rats analyzed by in vivo micro-CT. J Orthop Res 28:62-69 18.

Sehmisch S, Galal R, Kolios L, et al. (2009) Effects of low-magnitude, high-frequency mechanical stimulation in the rat osteopenia model. Osteoporos Int a J Establ as result Coop between Eur Found Osteoporos Natl Osteoporos Found USA 20:1999-2008 19.

Rubinacci A, Marenzana M, Cavani F et al (2008) Ovariectomy sensitizes rat cortical bone to whole-body vibration. Calcif Tissue Int 82:316-326

20.

Bouxsein ML, Boyd SK, Christiansen BA et al (2010) Guidelines for assessment of bone microstructure in rodents using micro-computed tomography. J Bone Miner Res Off J Am Soc Bone Miner Res 25:1468-1486 


\section{1.}

Adams JE (2013) Advances in bone imaging for osteoporosis. Nat Rev Endocrinol 9:28-42. doi:10.1038/nrendo.2012.217

22.

Khosla S, Oursler MJ, Monroe DG (2012) Estrogen and the skeleton. Trends Endocrinol Metab TEM 23:576-581. doi:10.1016/j.tem.2012.03.008

23.

Ishihara A, Sasaki T, Debari K et al (1999) Effects of ovariectomy on bone morphology in maxillae of mature rats. J Electron Microsc (Tokyo) 48:465-469

24.

Shi H-F, Cheung W-H, Qin L et al (2010) Low-magnitude high-frequency vibration treatment augments fracture healing in ovariectomy-induced osteoporotic bone. Bone 46:1299-1305. doi: $\underline{10.1016 / j . b o n e .2009 .11 .028}$

25.

Van Der Jagt OP, Van Der Linden JC, Waarsing JH et al (2012) Low-magnitude whole body vibration does not affect bone mass but does affect weight in ovariectomized rats. J Bone Miner Metab 30:40-46. doi:10.1007/s00774-011-0293-5

26.

Burghardt AJ, Kazakia GJ, Sode M et al (2010) A longitudinal HR-pQCT study of alendronate treatment in postmenopausal women with low bone density: relations among density, cortical and trabecular microarchitecture, biomechanics, and bone turnover. J Bone Miner Res Off J Am Soc Bone Miner Res 25:2558-2571. doi:10.1002/jbmr.157 27.

Eastell R, Walsh JS, Watts NB, Siris E (2011) Bisphosphonates for postmenopausal osteoporosis. Bone 49:82-88. doi:10.1016/j.bone.2011.02.011

28.

Rogers MJ, Crockett JC, Coxon FP, Mönkkönen J (2011) Biochemical and molecular mechanisms of action of bisphosphonates. Bone 49:34-41 29.

Roschger P, Rinnerthaler S, Yates J et al (2001) Alendronate increases degree and uniformity of mineralization in cancellous bone and decreases the porosity in cortical bone of osteoporotic women. Bone 29:185-191

30.

Feher A, Koivunemi A, Koivunemi M et al (2010) Bisphosphonates do not inhibit periosteal bone formation in estrogen deficient animals and allow enhanced bone modeling in response to mechanical loading. Bone 46:203-207 
31.

Lee K, Jessop H, Suswillo R, et al. (2003) Endocrinology: bone adaptation requires oestrogen receptor-alpha. Nature 24;389

32.

Lee KCL, Lanyon LE (2004) Mechanical loading influences bone mass through estrogen receptor [alpha]. Exerc Sport Sci Rev 32:64-68

33.

Lim SK, Won YJ, Lee HC et al (1999) A PCR analysis of ERalpha and ERbeta mRNA abundance in rats and the effect of ovariectomy. J Bone Miner Res Off J Am Soc Bone Miner Res 14:1189-1196

34.

Hoyland J (1999) Effect of ovarian steroid deficiency on oestrogen receptor alpha expression in bone. J Pathol 188:294-303

35.

Seeman E (2007) The periosteum—a surface for all seasons. Osteoporos Int 18:123-128 36.

Saxon L, Turner C (2005) Estrogen receptor beta: the antimechanostat? Bone 36:185-192 37.

Saxon LK, Robling AG, Castillo AB et al (2007) The skeletal responsiveness to mechanical loading is enhanced in mice with a null mutation in estrogen receptor. Am J Physiol Endocrinol Metab 293:E484-E491. doi:10.1152/ajpendo.00189.2007

38.

Saxon LK, Turner CH (2006) Low-dose estrogen treatment suppresses periosteal bone formation in response to mechanical loading. Bone 39:1261-1267

39.

Iwamoto J, Takeda T, Sato Y, Uzawa M (2005) Effect of whole-body vibration exercise on lumbar bone mineral density, bone turnover, and chronic back pain in post-menopausal osteoporotic women treated with alendronate. Aging Clin Exp Res 17:157-163 40.

Iwamoto J, Sato Y, Takeda T, Matsumoto H (2012) Whole body vibration exercise improves body balance and walking velocity in postmenopausal osteoporotic women treated with alendronate: Galileo and Alendronate Intervention Trail (GAIT). J Musculoskelet Neuronal Interact 12:136-143

41. 
Lespessailles E, Jaffré C, Beaupied H et al (2009) Does exercise modify the effects of zoledronic acid on bone mass, microarchitecture, biomechanics, and turnover in ovariectomized rats? Calcif Tissue Int 85:146-157

42.

Fuchs R, Shea M, Durski S et al (2007) Individual and combined effects of exercise and alendronate on bone mass and strength in ovariectomized rats. Bone 41:290-296 43.

Waarsing JH, Day JS, Van Der Linden JC et al (2004) Detecting and tracking local changes in the tibiae of individual rats: a novel method to analyse longitudinal in vivo micro-CT data. Bone 34:163-169

44.

Boyd SK, Davison P, M??ller R, Gasser JA (2006) Monitoring individual morphological changes over time in ovariectomized rats by in vivo micro-computed tomography. Bone 39:854-862

45.

Pei L, Tontonoz P (2004) Fat's loss is bone’s gain. J Clin Invest 113:805-806 